\title{
Greedy Multiprocessor Server Scheduling
}

\author{
Carl Bussema* and Eric Torng ${ }^{\dagger}$ \\ Department of Computer Science and Engineering, Michigan State University
}

\begin{abstract}
We show that the greedy Highest Density First (HDF) algorithm is $(1+$ $\epsilon)$-speed $O(1)$-competitive for the problem of minimizing the $\ell_{p}$ norms of weighted flow time on $m$ identical machines. Similar results for minimizing unweighted flow provide insight into the power of migration.
\end{abstract}

Keywords: online scheduling, algorithms, flow, multiprocessor, $\ell_{p}$ norm, greedy

\section{Introduction}

Server scheduling is a difficult problem with many conflicting criteria. The most obvious criteria to use for evaluating a server is the total flow/wait/response time metric where the flow time of a job is its completion time minus its release time. However, algorithms that are optimal for minimizing total flow time often starve individual jobs which is undesirable. The problem becomes more complicated when we consider that jobs may have different priorities; for example jobs affected by nice on a Unix system. In a recent paper [5], Bansal and Pruhs persuasively argue that these conflicting criteria can be formalized as the weighted $\ell_{p}$ norms of flow. However, they consider only the uniprocessor server scheduling problem. The multiprocessor setting is important as enterprise web and file servers often are composed of multiple machines in order to handle the large volume of requests they need to serve. In this paper, we show that the Highest Density First (HDF) algorithm is an effective algorithm for the multiprocessor server scheduling problem. A key step in our proof is showing that the Shortest Job First (SJF) and Shortest Remaining Processing Time (SRPT) algorithms effectively at minimizing the unweighted $\ell_{p}$ norms of flow and stretch on multiple machines.

\subsection{Formal Definition of Problem}

We will use the following notation and definitions throughout the paper. An input instance $I$ consists of $n$ jobs where job $J_{i}$ is characterized by its release time, $r_{i}$, its weight or priority $w_{i}$, and its size or work $p_{i}$. The density of a job $d_{i}=w_{i} / p_{i}$. The jobs will be scheduled on $m$ identical machines where no job can be assigned to more than one machine at any time. We assume that jobs can be preempted and migrated

\footnotetext{
*bussemac@cse.msu.edu

†Supported in part by NSF grant CCR 0105283. Corresponding author: Eric Torng, 3115 Engineering Building, Department of Computer Science and Engineering, Michigan State University, East Lansing, MI 48824, torng@cse.msu.edu.
} 
from one machine to another with no penalty. Finally, the remaining size of a job at any time $t$ is its initial size minus the amount of work done on that job up to time $t$.

Given a scheduling algorithm $A$, we denote the completion time of job $J_{i}$ as $c_{i}(A)$. The flow time of job $J_{i}$, denoted $F_{i}(A)$, is $c_{i}(A)-r_{i}$. The stretch of job $J_{i}$, denoted $S_{i}(A)$, is $F_{i}(A) / p_{i}$. We define $F^{p}(A, I)=\sum_{i}\left(F_{i}(A)\right)^{p}, S^{p}(A, I)=\sum_{i}\left(S_{i}(A)\right)^{p}$, and $W F^{p}(A, I)=\sum_{i} w_{i}\left(F_{i}(A)\right)^{p}$. The $\ell_{p}$ norms of flow, stretch, and weighted flow are $\left(F^{p}(A, I)\right)^{1 / p},\left(S^{p}(A, I)\right)^{1 / p}$, and $\left(W F^{p}(A, I)\right)^{1 / p}$, respectively. To simplify notation, we often drop the $A$ and $I$ when the algorithm or input instance is clear from context.

An online algorithm only becomes aware of a job after its release time while an offline algorithm knows the entire input instance immediately. We consider only clairvoyant algorithms that know the size (and weight) of a job as soon as it is released. Nonclairvoyant algorithms only learn the size of a job when it completes. We use OPT to denote an algorithm that computes the optimal schedule for any input instance.

Let $M(A(I))$ denote the cost of algorithm A when applied to input instance $I$ when we focus on metric $M$ where $M$ may be the $\ell_{p}$ norm of flow, stretch, or weighted flow. An online algorithm $A$ is said to be c-competitive for some metric $M$ if $M(A(I)) \leq$ $c \cdot M(O P T(I))$ for any input instance $I$. An algorithm $A$ using resource augmentation in the form of faster processors is said to be $s$-speed $c$-competitive if the competitive ratio $c$ can be maintained when $A$ 's processors are $s$ times as fast as OPT's (note that $c$ may be defined in terms of $s$ and is not necessarily constant). Analogously, an algorithm $A$ is said to be $s$-machine $c$-competitive if the competitive ratio $c$ can be maintained when $A$ has $s$ times as many processors as OPT.

\subsection{Definition of Algorithms}

SJF, SRPT, and HDF are greedy, preemptive, migratory algorithms that always schedule the $m$ unfinished jobs with highest priority where the priority metric differs for each algorithm. For SJF, the priority is the initial size of the job, $p_{i}$. For SRPT, the priority is the remaining size of the job. For HDF, the priority is the density of the job, $d_{i}$. In all algorithms, ties are broken first in favor of a running job, then arbitrarily. Furthermore, jobs are held in a central queue until assigned to a machine, and a preempted job is returned to the queue.

\subsection{Previous Results and Related Work}

Bansal and Pruhs showed that no online algorithm can be constant competitive for the problem of minimizing the $\ell_{p}$ norms of flow or stretch for $2 \leq p<\infty$, even in the uniprocessor environment [4]. Thus, to achieve constant competitive ratios for the problem of minimizing the weighted $\ell_{p}$ norm of flow, we are forced to consider the resource augmentation technique popularized by Kalyanasundaram and Pruhs [10]. Bansal and Pruhs show that several algorithms including SJF and SRPT are $(1+\epsilon)$ speed $O(1)$-competitive algorithms for minimizing the $\ell_{p}$ norms of flow and stretch on a single machine [4]. Chekuri et al. then show that simple uniprocessor scheduling algorithms such as SRPT combined with simple load balancing algorithms are also $(1+\epsilon)$-speed $O(1)$-competitive for minimizing the $\ell_{p}$ norms of flow and stretch on $m$ identical machines [8]. In particular, they consider an algorithm IMD that immediately dispatches jobs to machines and allows no migrations introduced by Avrahami and Azar [1]. The load balancer for IMD divides jobs into classes according to their size. It then assigns jobs to machines applying the greedy list scheduling approach; namely, a machine which has received the least total load of jobs in the given class is assigned 
the newly arrived job. Bansal and Pruhs then show that HDF and a nonclairvoyant algorithm are $(1+\epsilon)$-speed $O(1)$-competitive for the problem of minimizing weighted $\ell_{p}$ norms of flow on a single machine [5].

If we ignore the $\ell_{p}$ norms and focus only on total flow, stretch, or weighted flow, more results are known. On a single machine, Bansal and Dhamdhere give an $O(\log W)$ algorithm for minimizing weighted flow time where $W$ is the ratio of the maximum job weight divided by the minimum job weight [3]. This algorithm is constant competitive if there are only a constant number of distinct job weights. With resource augmentation, Becchetti et al. show that HDF is $(1+\epsilon)$-speed $O(1+1 / \epsilon)$-competitive in the uniprocessor setting and $(2+\epsilon)$-speed $O(1+1 / \epsilon)$-competitive in the multiprocessor setting [7]. Phillips et al. show that an algorithm they named Preemptively-ScheduleBy-Halves is a 2-speed 1-competitive algorithm on a single processor [14], and Becchetti et al. observe that this analysis also applies to HDF [7]. Bansal and Dhamdere then show that a nonclairvoyant algorithm is also $(1+\epsilon)$-speed $O(1+1 / \epsilon)$-competitive on a single machine matching HDF's performance [3].

If we consider total flow time, SRPT is an optimal algorithm in the uniprocessor setting, and Leonardi and Raz showed that SRPT is $O(\min \{\log n / m, \log P\})$ competitive in the multiprocessor setting where $P$ is the ratio of the maximum to minimum processing times of any jobs [11]. They further show that this is optimal within constant factors for any online algorithm, randomized or deterministic. Muthukrishnan et al. show that SRPT is constant competitive for minimizing stretch for the multiprocessor environment [13]. Many algorithms without migration and then with immediate dispatch were developed that had essentially the same competitive ratios within constant factors $[1,2,6,9]$. With resource augmentation, Phillips et al. first showed that SRPT is a $(2-1 / m)$-speed 1-competitive for minimizing total flow time on $m$ identical machines [14], and McCullough and Torng improved this result to show that SRPT is in fact an $s$-speed $1 / s$-competitive algorithm for minimizing total flow time on $m$ identical machines when $s \geq 2-1 / m[12]$. For more details on these and other results, consult the recent survey on online scheduling [15].

\subsection{Our Results}

We first extend the uniprocessor results of Bansal and Pruhs [4] by showing that SJF and SRPT are $(1+\epsilon)$-speed $O(1+1 / \epsilon)$-competitive for minimizing the $\ell_{p}$ norms of flow and stretch in the multiprocessor setting. These results also apply with extra machines. We then use our SJF extra speed result for flow time to show that HDF is a $(1+\epsilon)$-speed $O\left(1+1 / \epsilon^{2}\right)$-competitive for minimizing the weighted $\ell_{p}$ norms of flow in the multiprocessor setting, extending the uniprocessor result of Bansal and Pruhs [5]. While our SJF and SRPT results are comparable to those obtained by Chekuri et al. for IMD [8], we analyze conceptually simpler greedy algorithms than IMD. We also achieve smaller constants due to allowing migration. We use many techniques originally developed in $[4,8,5]$.

\section{SJF and SRPT}

We first define the following notation. Let $A$ denote any algorithm and $t$ denote any time. Let $U(A, t)$ be the set of unfinished jobs for algorithm $A$ at time $t$, or the number of such jobs. The total flow time of a schedule can be computed as $\int U(A, t) d t$. For any job $J_{i}$ in $U(A, t)$, let $A g e_{i}(t)=t-r_{i}$ and $S A g e_{i}(t)=A g e_{i}(t) / p_{i}$. Let $\operatorname{Age}^{p}(A, t)$ 
denote the sum over all jobs $J_{i} \in U(A, t)$ of $A g e_{i}(t)^{p-1}$, and let $S A g e^{p}(A, t)$ denote the sum over all jobs $J_{i} \in U(A, t)$ of $S A g e_{i}(t)^{p-1}$. Bansal and Pruhs observed that $F^{p}(A)=\int A g e^{p}(A, t) d t$ and that $S^{p}(A)=\int S A g e^{p}(A, t) d t[4]$.

We use $V_{\leq k}(t)$ to denote the sum of remaining sizes of all unfinished jobs of initial size at most $k$ for SJF or SRPT at time $t$ released prior to time $t$. We similarly define $V_{<k}^{*}(t)$ for OPT. We use $P_{\leq k}(t)$ to denote the sum of initial sizes of all unfinished jobs of initial size at most $k$ for SJF or SRPT at time $t$ released prior to time $t$. We use $P_{\leq k}\left(t_{1}, t_{2}\right)$ to denote the sum of initial sizes of all jobs of initial size at most $k$ released during interval $\left[t_{1}, t_{2}\right)$.

\subsection{Proof Structure}

Our strategy for proving competitive ratios for SJF and SRPT will follow that of [8] which is a modification of the uniprocessor strategy of [4]. We focus on the $\ell_{p}$ norms of flow time as the proof for stretch is essentially identical.

Given that $F^{p}(A)=\int A g e^{p}(A, t) d t$, we would prove our result if we can bound $A g e^{p}(A, t)$ relative to $\operatorname{Age}^{p}(O P T, t)$ for all times $t$. Unfortunately, as observed in [8], this is not possible as online algorithms can "fall behind" OPT so that there can be times where $\operatorname{Age}^{p}(A, t)>0$ while $\operatorname{Age}^{p}(O P T, t)=0$. To compensate for this problem, at any time $t$, we restrict our attention to unfinished jobs that are sufficiently old as was done in [8]. Formally, we define $U^{\alpha}(A, t)=\left\{J_{i} \in U(A, t) \mid A g e_{i}(t) \geq \alpha p_{i}\right\}$ where $\alpha>0$ is a constant to be defined later. We then define $\operatorname{Age}^{p}(A, t, \alpha)$ to be the sum over all jobs $J_{i} \in U^{\alpha}(A, t)$ of $A g e_{i}(t)^{p-1}$ and $F^{p}(A, \alpha)=\int A g e^{p}(A, t, \alpha) d t$. Finally, we define $D I F(A)=F^{p}(A)-F^{p}(A, \alpha)$. We can then bound $F^{p}(A)$ relative to $F^{p}(O P T)$ if we can individually bound both $F^{p}(A, \alpha)$ and $D I F(A)$ relative to $F^{p}(O P T)$.

We first observe that $F^{p}(O P T) \geq\left(\sum p_{i}^{p}\right)^{1 / p}$ as each job must be in the system for at least $p_{i}$ time while $D I F(A) \leq\left(\sum\left(\alpha p_{i}\right)^{p}\right)^{1 / p}$ as $F^{p}(A, \alpha)$ accounts for the entire time that each job is in the system except for at most the first $\alpha p_{i}$ time units. Thus, $D I F(A) \leq \alpha F^{p}(O P T)$.

\subsection{An Association Scheme for Bounding $F^{p}(A, \alpha)$}

For $F^{p}(A, \alpha)$, we observe that for any time $t$, jobs that are in both $U^{\alpha}(A, t)$ and $U(O P T, t)$ contribute equally to $\operatorname{Age}^{p}(A, t, \alpha)$ and $A g e^{p}(O P T, t)$. Thus, $A g e^{p}(A, t, \alpha)$ may greatly exceed $\operatorname{Age}^{p}(O P T, t)$ because of the jobs that are in $U^{\alpha}(A, t)$ but not in $U(O P T, t)$. We define $D(t)$ as $U^{\alpha}(A, t)-U(O P T, t)$ where $A$ is either SJF or SRPT; the specific algorithm will always be clear from context. $D(t)$ represents the old jobs that $A$ has left to finish at time $t$ but which OPT has already completed. We then define $A g e^{p}(D, t)$ to be the sum over all jobs $J_{i} \in D(t)$ of $A g e_{i}(t)^{p-1}$ and observe that $\operatorname{Age}^{p}(A, t, \alpha) \leq A g e^{p}(O P T, t)+\operatorname{Age}^{p}(D, t)$.

We now define an association scheme $S_{\beta}$ similar to that of [4] to show that $\operatorname{Age}^{p}(D, t)$ is bounded relative to $\operatorname{Age}^{p}(O P T, t)$. Given $0<\beta<1$, for each job $J_{j}$ in $U(O P T, t)$, we define a set of jobs $S_{\beta}(j)$ with the following properties:

1. For all $J_{i} \in S_{\beta}(j), \operatorname{Age}_{i}(t) \leq 1 / \beta A g e_{j}(t)$.

2. For all $J_{i} \in S_{\beta}(j), p_{j} \leq p_{i}$. With this constraint, the argument easily extends to $\ell_{p}$ norms of stretch as well.

3. $\left|S_{\beta}(j)\right| \leq 1 / \beta$.

4. $D(t)=\bigcup_{J_{j} \in U(O P T, t)} S_{\beta}(j)$ 
Assuming we can create such an association scheme $S_{\beta}$ for all times $t$, it follows that $\operatorname{Age}^{p}(D, t) \leq(1 / \beta)^{p} \operatorname{Age}^{p}(O P T, t)$ and thus $F^{p}(A, \alpha) \leq(1+1 / \beta) F^{p}(O P T)$. Combining with the earlier bound for $\operatorname{DIF}(A)$, we get that $F^{p}(A) \leq(1+\alpha+1 / \beta) F^{p}(O P T)$.

We now prove that an association scheme $S_{\beta}$ exists for all times $t$ and $\beta$ sufficiently large relative to $\epsilon$. For any time $t$, index the jobs in $D(t)$ in increasing order of size $p_{i}$. Given time $t$ and $i \leq|D(t)|$, define $t(i)=t-\beta\left(t-r_{i}\right)$. To prove that an association scheme $S_{\beta}$ exists, it sufficient to prove that we can always allocate to $J_{i} \in D(t)$ a $\beta p_{i}$ amount of work from $V_{\leq p_{i}}^{*}(t)$ from jobs with release time at most $t(i)$ that was not already allocated to a lower indexed job in $D(t)$.

Let $A V(t, i)$ denote the amount of work from $V^{*}(t)$ that can be assigned to the first $i$ jobs in $D(t)$, and let $W(t, i)$ denote the amount of work that must be allocated to the first $i$ jobs in $D(t)$. Our goal is to show that for any time $t$, any $i \leq|D(t)|$ and appropriate values of $\alpha$ and $\beta$ that $W(t, i) \leq A V(t, i)$.

Lemma 2.1 For any time $t$ and $i \leq|D(t)|, A V(t, i) \geq V_{\leq p_{i}}^{*}\left(r_{i}\right)+P_{\leq p_{i}}\left(r_{i}, t(i)\right)-$ $m\left(t-r_{i}\right)$.

Proof: OPT's unfinished work at time $t$ for jobs of length at most $p_{i}$ released before $t(i)$ is at least: OPT's unfinished work of jobs of this length as of $r_{i}$ plus the total work to be done for jobs of this length arriving in $\left[r_{i}, t(i)\right)$ minus the maximal work done by OPT during $\left[r_{i}, t\right)$.

Lemma 2.2 For any time $t$ and any $i \leq|D(t)|$,

$$
W(t, i) \leq \beta\left(m\left(t-r_{i}\right)+P_{\leq p_{i}}\left(r_{i}\right)\right)
$$

Proof: Consider the first $i$ jobs in $D(t)$. Some of these jobs are released at time $r_{i}$ or later. By definition of $D(t)$, OPT must finish these jobs by time $t$, so their total size can be at most $m\left(t-r_{i}\right)$. The other jobs must be released before time $r_{i}$ and are unfinished by $A$ at time $r_{i}$. Thus we can bound their processing times by $P_{\leq p_{i}}\left(r_{i}\right)$ and the result follows.

Theorem 2.1 Consider the online algorithms SJF and SRPT augmented with either $m(1+\epsilon)$-speed processors or $(1+\epsilon) m$ 1-speed processors. For any time $t$ and any $1 \leq i \leq|D(t)|$, there exist values of $\beta$ and $\alpha$ such that:

$$
(1+\beta) m\left(t-r_{i}\right)+\beta P_{\leq p_{i}}\left(r_{i}\right) \leq V_{\leq p_{i}}^{*}\left(r_{i}\right)+P_{\leq p_{i}}\left(r_{i}, t(i)\right)
$$

Corollary 2.1 SJF and SRPT are $(1+\epsilon)$-speed $O(1 / \epsilon)$-competitive and $(1+\epsilon) m$ machine $O(1 / \epsilon)$-competitive.

Proof: Our proofs in the next sections show that the following values for $\beta$ and $\alpha$ make Theorem 2.1 true. For SJF augmented with $(1+\epsilon)$-speed processors, $\beta=\frac{\epsilon}{6(1+\epsilon)}$ and $\alpha=\frac{4}{\epsilon}+4$; for SRPT with $(1+\epsilon)$-speed, $\beta=\frac{\epsilon}{6(1+\epsilon}$ and $\alpha=\frac{6}{\epsilon}+6$; for SJF with $(1+\epsilon) m$ machines, $\beta=\frac{\epsilon}{6(1+\epsilon)}$ and $\alpha=\frac{4}{\epsilon}+8+6 \epsilon$; and for SRPT with $(1+\epsilon) m$ machines, $\beta=\frac{\epsilon}{6(1+\epsilon)}$ and $\alpha=\frac{6}{\epsilon}+12+6 \epsilon$.

We prove Theorem 2.1 in the next sections, first considering SJF with faster machines. 


\section{$2.3 \quad$ SJF}

We seek to prove Theorem 2.1 for SJF and faster machines. First, we derive a bound on $V_{\leq k}^{*}$, the volume of remaining work from jobs of initial size at most $k$ for OPT at any time $u$. This derivation requires new arguments than those from $[4,8]$. In [8], because of the properties of IMD, their analysis uses the earliest time point $u^{\prime}$ where some machine processes jobs of size at most $k$ continuously in the interval $\left(u^{\prime}, u\right]$. This time point will not work for SJF (or SRPT). Instead, we identify the latest time point $u^{\prime}<u$ where some machine is not processing a job of size at most $k$.

Lemma 2.3 When SJF is given $(1+\epsilon)$-speed processors, for any time $u$ and any job size $k$,

$$
V_{\leq k}^{*}(u) \geq \frac{\epsilon}{1+\epsilon} P_{\leq k}(u)+\frac{1}{1+\epsilon} V_{\leq k}(u)-m k
$$

Proof: We first focus on SJF's work. Define time $u^{\prime}<u$ as the most recent time (possibly 0) such that for some positive interval of time just prior to $u^{\prime}$, SJF had an idle machine or was running a job with initial size $>k$. By definition of $u^{\prime}$, SJF has at most $m-1$ unfinished jobs of initial size at most $k$ at time $u^{\prime}$ that were released prior to time $u^{\prime}$. Thus, $V_{\leq k}\left(u^{\prime}\right) \leq(m-1) k<m k$. By the definition of $u^{\prime}$, every machine must remain busy until $u$ working on jobs with initial size at most $k$. Thus, SJF does at least $(1+\epsilon) m$ work on jobs of initial size at most $k$ during the interval $\left[u^{\prime}, u\right)$. Finally, the amount of work of initial size at most $k$ that arrives during the interval $\left[u^{\prime}, u\right)$ is $P_{\leq k}\left(u^{\prime}, u\right)$. This leads to the following inequality:

$$
V_{\leq k}(u) \leq m k+P_{\leq k}\left(u^{\prime}, u\right)-(1+\epsilon) m\left(u-u^{\prime}\right)
$$

which can be rewritten as $-m\left(u-u^{\prime}\right) \geq \frac{1}{1+\epsilon}\left(V_{\leq k}(u)-m k-P_{\leq k}\left(u^{\prime}, u\right)\right)$.

When we look at OPT, it is clear that $V_{\leq k}^{*}(u) \geq P_{\leq k}\left(u^{\prime}, u\right)-m\left(u-u^{\prime}\right)$. Combining with our previous inequality yields yields $V_{\leq k}^{*}(u) \geq \frac{\epsilon}{1+\epsilon} P_{\leq k}\left(u^{\prime}, u\right)+\frac{1}{1+\epsilon} V_{\leq k}(u)-$ $\frac{1}{1+\epsilon} m k$. To complete the proof, we then observe that $P_{\leq k}\left(u^{\prime}, u\right) \geq P_{\leq k}(u)-m k$, as at most $m k$ of the unfinished work for SJF at time $u$ could have come prior to time $u^{\prime}$, by its definition.

From here, we use (2) to substitute into (1), changing variables $\left(u=r_{i}\right.$ and $\left.k=p_{i}\right)$. We then replace $P_{\leq p_{i}}\left(r_{i}\right)$ with $V_{\leq p_{i}}\left(r_{i}\right)$ which is obviously smaller. Combining like terms and restricting $\beta \leq \frac{\epsilon}{1+\epsilon}$ gives the following goal.

$$
(1-\beta) V_{\leq p_{i}}\left(r_{i}\right)+P_{\leq p_{i}}\left(r_{i}, t(i)\right) \geq(1+\beta) m\left(t-r_{i}\right)+m p_{i}
$$

We now derive a lower bound on $V_{\leq p_{i}}\left(r_{i}\right)+P_{\leq p_{i}}\left(r_{i}, t(i)\right)$ by deriving a lower bound on the work done by SJF during $\left[r_{i}, t(i)\right)$ on jobs of length at most $p_{i}$.

Lemma 2.4 For any job $J_{i} \in D(t)$, we derive the following bound:

$$
V_{\leq p_{i}}\left(r_{i}\right)+P_{\leq p_{i}}\left(r_{i}, t(i)\right) \geq(1+\epsilon) m\left(t(i)-r_{i}-p_{i} /(1+\epsilon)\right)
$$

Proof: We observe that if SJF works on $J_{i}$ for $p_{i} /(1+\epsilon)$ time in $\left[r_{i}, t(i)\right)$, it finishes $J_{i}$ by time $t(i)$, thus $J_{i}$ would not be in $D(t)$. So SJF uses all machines to work on jobs of size at most $p_{i}$ for at least $t(i)-r_{i}-\frac{p_{i}}{1+\epsilon}$ time in $\left[r_{i}, t(i)\right)$. These jobs must have been in $U\left(r_{i}\right)$ or arrived during $\left[r_{i}, t(i)\right)$ and the result follows. 
Using (5) to substitute into (4) (noting that $t(i)-r_{i}=(1-\beta)\left(t-r_{i}\right)$ ), we get:

$$
(1-\beta)\left((1+\epsilon)(1-\beta) m\left(t-r_{i}\right)-m p_{i}\right) \geq(1+\beta) m\left(t-r_{i}\right)+m p_{i}
$$

To complete the proof, we need to select values for $\beta$ and $\alpha$. Intuitively, returning to the association scheme $S_{\beta}$, we are trying to associate unfinished jobs from OPT with jobs in $D(t)$. Increasing $\alpha$ helps by restricting the membership of $D(t)$. Decreasing $\beta$ helps as each job in OPT can be associated with more jobs in $D(t)$. We now derive specific values of $\beta$ and $\alpha$ that imply (6).

Since we have previously restricted $\beta \leq \frac{\epsilon}{1+\epsilon}$, we will start by selecting $\beta=\frac{\epsilon}{c(1+\epsilon)}$, where $c$ is a constant $\geq 1$ to be determined later. Substituting this into the above, we get the following sequence of goals.

$$
\begin{aligned}
\frac{(c+c \epsilon-\epsilon)^{2}}{c^{2}(1+\epsilon)}\left(t-r_{i}\right)-\frac{c+c \epsilon+\epsilon}{c(1+\epsilon)}\left(t-r_{i}\right) & \geq(2-\beta) p_{i} \\
\left(\frac{c^{2} \epsilon-3 c \epsilon+c^{2} \epsilon^{2}-2 c \epsilon^{2}+\epsilon^{2}}{c^{2}}\right)\left(t-r_{i}\right) & \geq 2(1+\epsilon) p_{i} \\
\left(\frac{c^{2} \epsilon-3 c \epsilon}{c^{2}}\right)\left(t-r_{i}\right) & \geq 2(1+\epsilon) p_{i} \\
\left(t-r_{i}\right) & \geq\left(\frac{2 c+2 c \epsilon}{(c-3) \epsilon}\right) p_{i}
\end{aligned}
$$

Note that the 3rd goal implies the second goal when $c \geq 2$ as $c^{2} \epsilon^{2}-2 c \epsilon^{2}+\epsilon^{2} \geq 0$ when $c \geq 2$. We can satisfy the last goal as long as we choose $\alpha=(2 c+2 c \epsilon) /((c-3) \epsilon)$. We now choose a value of $c$ that minimizes $(1+1 / \beta+\alpha)$. For SJF, selecting $c=6$ yields $\beta=\frac{\epsilon}{6(1+\epsilon)}$ and $\alpha=\frac{4}{\epsilon}+4$ giving a total competitive ratio of $\frac{10}{\epsilon}+11=O\left(\frac{1}{\epsilon}\right)$.

We now compare our bounds for SJF with bounds for IMD, the non-migratory loadbalanced algorithm described in [8]. There, $\beta$ was chosen to be $\frac{\epsilon}{4(1+\epsilon)}$, giving an $\alpha$ of $\frac{96}{\epsilon}$ for IMD with extra speed. A better bound can be achieved by selecting $\beta=\frac{\epsilon}{27(1+\epsilon)}$ to yield a competitive ratio of $\frac{54}{\epsilon}+\frac{110}{3}$. While neither bound is necessarily tight, this comparison gives us some insight into the power of migration for this problem.

\subsection{SRPT}

To modify our proof for SJF with faster machines to SRPT with faster machines, we need to prove the equivalent of Lemma 2.3 and Lemma 2.4 for SRPT.

Lemma 2.5 When SRPT is given $(1+\epsilon)$-speed processors, for any time $u$ and job size $k$,

$$
V_{\leq k}^{*}(u) \geq(\epsilon /(1+\epsilon)) P_{\leq k}(u)+(1 /(1+\epsilon)) V_{\leq k}(u)-m k
$$

Proof: We first focus on SRPT and define time $u^{\prime}<u$ similar to before: the most recent time (possibly 0) such that for some positive interval of time ending at $u^{\prime}$, SRPT had an idle machine or was running a job with remaining size $>k$. From this, there can be at most $m$ unfinished jobs released prior to time $u^{\prime}$ with remaining size at most $k$ at time $u^{\prime}$. Let $m_{1}$ of these jobs have $p_{i}>k$ while the other $m_{2} \leq m-m_{1}$ jobs have $p_{i} \leq k$, making $V_{\leq k}\left(u^{\prime}\right) \leq m_{2} k$.

By the definition of $u^{\prime}$, every machine in the interval $\left[u^{\prime}, u\right)$ must be busy processing jobs of remaining size at most $k$. In particular, any jobs that arrive in the interval $\left[u^{\prime}, u\right)$ that are processed must have initial size at most $k$, so the only work done during 
the interval $\left[u^{\prime}, u\right)$ on jobs with initial size larger than $k$ is any work done on the $m_{1}$ unfinished jobs at time $u^{\prime}$. SRPT can do at most $m_{1} k$ work on these jobs during $\left[u^{\prime}, u\right)$. Thus, SRPT does at least $(1+\epsilon) m-m_{1} k$ work on jobs of initial size at most $k$ during $\left[u^{\prime}, u\right)$. This leads to the following: $V_{\leq_{k}}(u) \leq m k+P_{\leq k}\left(u^{\prime}, u\right)-(1+\epsilon) m\left(u-u^{\prime}\right)$. It is still clear that $V_{\leq k}^{*}(u) \geq P_{\leq k}\left(u^{\prime}, u\right)-m\left(u-u^{\prime}\right)$. Combine the above inequalities and the same observation that $P_{\leq k}\left(u^{\prime}, u\right) \geq P_{\leq k}(u)-m k$ to complete the proof.

Lemma 2.6 For any job $J_{i} \in D(t)$, we derive the following bound:

$$
V_{\leq k}\left(r_{i}\right)+P_{\leq k}\left(r_{i}, t(i)\right) \leq(1+\epsilon) m\left(t(i)-r_{i}-\left(p_{i} /(1+\epsilon)\right)\right)-m p_{i} .
$$

Proof Sketch: The main change from the proof for SJF is that it is possible SRPT does $m p_{i}$ work on jobs with initial size $>p_{i}$ but remaining size at most $p_{i}$ during interval $\left[r_{i}, t(i)\right)$. Thus we must subtract an additional $m p_{i}$ term.

Finishing the proof the same way as for SJF, we select $\beta=\frac{\epsilon}{6(1+\epsilon)}$ and $\alpha=\frac{6}{\epsilon}+6$, for a ratio of $\frac{12}{\epsilon}+13=O\left(\frac{1}{\epsilon}\right)$.

\subsection{Extra machines}

To modify our proofs of SJF and SRPT with faster machines to SJF and SRPT with extra machines, we need to prove the equivalent of Lemma 2.3 and Lemma 2.4 for both SJF and SRPT.

Lemma 2.7 When SJF or SRPT is given $(1+\epsilon) m$ speed-1 processors, for any time $u$ and job size $k$,

$$
V_{\leq k}^{*}(u) \geq \frac{\epsilon}{1+\epsilon} P_{\leq k}(u)+\frac{1}{1+\epsilon} V_{\leq k}(u)-(1+\epsilon) m k .
$$

Proof Sketch: The key change for this proof is that the work SJF and SRPT have left at any time $u$ is increased slightly. Specifically, at time $u^{\prime}$, we can only assume that $V_{\leq k}\left(u^{\prime}\right) \leq(1+\epsilon) m k$ instead of $V_{\leq k}\left(u^{\prime}\right) \leq m k$. Because of this, we need to replace inequality (3) with $V_{\leq k}(u) \leq(1+\epsilon) m k+P_{\leq k}\left(u^{\prime}, u\right)-(1+\epsilon) m\left(u-u^{\prime}\right)$ and we must use $P_{\leq k}\left(u^{\prime}, u\right) \geq P_{\leq k}(u)-(1+\epsilon) m k$ where we previously used $P_{\leq k}\left(u^{\prime}, u\right) \geq P_{\leq k}(u)-m k$.

We now prove modified versions of Lemmas 2.4 and 2.6.

Lemma 2.8 Consider SJF. For any time $t$ and any job $J_{i} \in D(t)$, we derive the following bound:

$$
V_{\leq p_{i}}\left(r_{i}\right)+P_{\leq p_{i}}\left(r_{i}, t(i)\right) \geq(1+\epsilon) m\left(t(i)-r_{i}-p_{i}\right) .
$$

Proof Sketch: The key change is that SJF could not have worked on job $J_{i}$ during $\left[r_{i}, t(i)\right)$ for $p_{i}$ time units (instead of $p_{i} /(1+\epsilon)$ time units) or else $J_{i}$ would be completed at time $t$ and thus not part of $D(t)$. Thus, SJF must devote all $(1+\epsilon) m$ machines for at least $t(i)-r_{i}-p_{i}$ time units to other jobs of size at most $p_{i}$ during the interval $\left[r_{i}, t(i)\right)$. 
Lemma 2.9 Consider SRPT. For any time $t$ and any job $J_{i} \in D(t)$, we derive the following bound:

$$
V_{\leq k}\left(r_{i}\right)+P_{\leq k}\left(r_{i}, t(i)\right) \geq(1+\epsilon) m\left(t(i)-r_{i}-2 p_{i}\right) .
$$

Proof Sketch: Like the above proof, we now must assume SRPT could not have worked on job $J_{i}$ for $p_{i}$ time units instead of $p_{i} /(1+\epsilon)$ time units. Furthermore, $(1+\epsilon) m p_{i}$ of the work that SRPT does on other jobs might come from jobs that have initial sizes larger than $p_{i}$.

Putting everything back together, for SJF, we select $\beta=\frac{\epsilon}{6(1+\epsilon)}$ and $\alpha=\frac{4}{\epsilon}+8+6 \epsilon$, so the total ratio is $\frac{10}{\epsilon}+15+6 \epsilon=O\left(\frac{1}{\epsilon}\right)$. For SRPT, we select $\beta=\frac{\epsilon}{6(1+\epsilon)}$ and $\alpha=\frac{6}{\epsilon}+12+6 \epsilon$, giving a ratio of $\frac{12}{\epsilon}+19+6 \epsilon=O\left(\frac{1}{\epsilon}\right)$. These compare to the best values for $I M D$ with extra machines in [8] of $\beta=\frac{\epsilon}{27(1+\epsilon)}$ and $\alpha=\frac{27}{\epsilon}+\frac{80}{3}$, for a total ratio of $\frac{54}{\epsilon}+\frac{164}{3}$.

\section{HDF}

We now consider the weighted $\ell_{p}$ norms of flow. We follow the proof technique used in [5] for a single machine, extending it now for multiple machines relying on our multiprocessor result for SJF for unweighted flow. We adopt their notations of $O P T(M, \mathcal{I}, S)$ as meaning the value of the optimal solution for metric $M$ on input instance $\mathcal{I}$ given $S$-speed processors, and $M(A, \mathcal{I}, S)$ as meaning the worst-case solution for metric $M$ under algorithm $A$ on input instance $\mathcal{I}$ with $S$-speed processors.

Given an input instance $\mathcal{I}$, we define $\mathcal{I}^{\prime}$ as follows: for each $J_{i} \in \mathcal{I}$, create $w_{i}$ identical jobs in $\mathcal{I}^{\prime}$, each of size $p_{i} / w_{i}$, weight 1 , and release time $r_{i}$. Denote these as $J_{i_{1}}^{\prime}, J_{i_{2}}^{\prime}, \ldots J_{i_{w_{i}}}^{\prime}$ and let $X_{i}=\left\{J_{i_{1}}^{\prime} \ldots J_{i_{w_{i}}}^{\prime}\right\}$.

Lemma 3.1 Given an input instance $\mathcal{I}$ and related instance $\mathcal{I}^{\prime}$ as defined above,

$$
O P T\left(F^{p}, \mathcal{I}^{\prime}, 1\right) \leq O P T\left(W F^{p}, \mathcal{I}, 1\right)
$$

Proof: Let $\mathcal{S}$ be a schedule which minimizes the $\ell_{p}$ norm of weighted flow for $\mathcal{I}$. Given $S$ we schedule $\mathcal{I}^{\prime}$ as follows: for any time $t$, for a given machine $a^{\prime}$ under $O P T\left(\mathcal{I}^{\prime}\right)$, work on a job in $X_{i}$ if and only if $J_{i}$ is being processed on the corresponding machine $a$ under $S$ at $t$.

Thus all jobs in $X_{i}$ are finished when $J_{i}$ finishes, so no job in $X_{i}$ has a higher flow than $J_{i}$, which by definition is $w_{i} f_{i}^{p}$. The $w_{i}$ jobs in $X_{i}$ each contribute a maximum of $f_{i}^{p}$ to flow, for a maximum total of $w_{i} f_{i}^{p}$, and $O P T$ can do no worse.

From Theorem 2.1, we know that SJF is $(1+\epsilon)$-speed $O(1 / \epsilon)$-competitive for $\ell_{p}$ norms of flow on multiple machines. Thus,

Fact 3.1

$$
F^{p}\left(S F J, \mathcal{I}^{\prime}, 1+\epsilon\right)=O\left(\frac{1}{\epsilon}\right) O P T\left(F^{p}, \mathcal{I}^{\prime}, 1\right)
$$

Lemma 3.2 For every job $J_{i} \in \mathcal{I}$ and time $t$, if $J_{i}$ is alive under HDF with speed $(1+\epsilon)$, at least $\frac{w_{i} \epsilon}{1+\epsilon}$ jobs in $X_{i} \in \mathcal{I}^{\prime}$ are alive at $t$ under $S J F$ with speed 1. 
Proof: Suppose $t$ is the earliest time when $J_{i}$ is alive under HDF and there are less than $\frac{w_{i} \epsilon}{1+\epsilon}$ jobs from $X_{i}$ alive under SJF. Since $J_{i}$ is alive under HDF with $(1+\epsilon)$-speed processors, HDF has spent less than $\frac{p_{i}}{1+\epsilon}$ time on $J_{i}$, whereas SJF has spent more than that amount of time on jobs in $X_{i}$.

So there must exist some time $t^{\prime} \in\left[r_{i}, t\right)$ when HDF was not running $J_{i}$ on any machine and SJF was processing at least one job in $X_{i}$ on some machine. Therefore, there exist $m$ jobs $J_{j_{1}} \ldots J_{j_{m}}$ with strictly higher density than $J_{i}$ being processed at $t^{\prime}$. Thus, the $X_{j_{1}} \ldots X_{j_{m}}$ jobs would all have smaller sizes than those in $X_{i}$. Since SJF works on $X_{i}$ at time $t^{\prime}$, it must have finished all the jobs in $X_{j_{1}} \ldots X_{j_{m}}$ by $t^{\prime}$, and since $J_{j_{1}} \ldots J_{j_{m}}$ are alive under HDF at $t^{\prime}$, this contradicts the assumption of the minimality of $t$.

Corollary 3.1 HDF is a $(1+\epsilon)$-speed $O\left(1 / \epsilon^{2}\right)$-competitive algorithm for minimizing the weighted $\ell_{p}$ norms of flow on $m$ identical machines.

Proof: We get $W F^{p}(H D F, \mathcal{I}, 1+\epsilon) \leq(1+1 / \epsilon)^{p} F^{p}\left(S J F, \mathcal{I}^{\prime}, 1\right)$ from Lemma 3.2. From Fact 3.1, we see that $W F^{p}(H D F, \mathcal{I}, 1+\epsilon)=O\left(\frac{1}{\epsilon^{2}}\right)^{p} O P T\left(W F^{p}, \mathcal{I}, 1\right)$, and the result follows.

\section{References}

[1] Nir Avrahami and Yossi Azar. Minimizing total flow time and total completion time with immediate dispatching. In Proc. 15th Symp. on Parallel Algorithms and Architectures (SPAA), pages 11-18. ACM, 2003.

[2] Baruch Awerbuch, Yossi Azar, Stefano Leonardi, and Oded Regev. Minimizing the flow time without migration. In Proc. 31st Symp. Theory of Computing (STOC), pages 198-205. ACM, 1999.

[3] Nikhil Bansal and K. Dhamdhere. Minimizing weighted flow time. In Proc. 14th Symp. on Discrete Algorithms (SODA), pages 508-516. ACM/SIAM, 2003.

[4] Nikhil Bansal and Kirk Pruhs. Server scheduling in the $L_{p}$ norm: A rising tide lifts all boats. In Proc. 35th Symp. Theory of Computing (STOC), pages 242-250. ACM, 2003.

[5] Nikhil Bansal and Kirk Pruhs. Server scheduling in the weighted $\ell_{p}$ norm. In Proc. 6th Theoretical Informatics, Latin American Symposium (LATIN), pages 434-443, 2004.

[6] L. Becchetti, S. Leonardi, and S. Muthukrishnan. Scheduling to minimize average stretch without migration. In Proc. 11th Symp. on Discrete Algorithms (SODA), pages 548-557. ACM/SIAM, 2000.

[7] Luca Becchetti, Stefano Leonardi, Alberto Marchetti-Spaccamela, and Kirk Pruhs. Online weighted flow time and deadline scheduling. In RANDOMAPPROX, volume 2129 of Lecture Notes in Computer Science, pages 36-47. Springer, 2001.

[8] Chandra Chekuri, Ashish Goel, Sanjeev Khanna, and Amit Kumar. Multiprocessor scheduling to minimize flow time with $\epsilon$ resource augmentation. In Proc. 36th Symp. Theory of Computing (STOC), pages 363-372. ACM, 2004. 
[9] Chandra Chekuri, Sanjeev Khanna, and An Zhu. Algorithms for weighted flow time. In Proc. 33rd Symp. Theory of Computing (STOC), pages 84-93. ACM, 2001.

[10] Bala Kalyanasundaram and Kirk Pruhs. Speed is as powerful as clairvoyance. Journal of the ACM, 47:214-221, 2000.

[11] Stefano Leonardi and Danny Raz. Approximating total flow time on parallel machines. In Proc. 29th Symp. Theory of Computing (STOC), pages 110-119. ACM, 1997.

[12] Jason McCullough and Eric Torng. SRPT optimally uses faster machines to minimize flow time. In Proc. 15th Symp. on Discrete Algorithms (SODA), pages 343-351. ACM/SIAM, 2004.

[13] S. Muthukrishnan, R. Rajaraman, A. Shaheen, and J. E. Gehrke. Online scheduling to minimize avarage strech. In Proc. 40th Symp. Foundations of Computer Science (FOCS), pages 433-443. IEEE, 1999.

[14] Cynthia Phillips, Cliff Stein, Eric Torng, and Joel Wein. Optimal time-critical scheduling via resource augmentation. Algorithmica, pages 163-200, 2002.

[15] Kirk Pruhs, Jiř́ Sgall, and Eric Torng. Online scheduling. In Joseph Leung, editor, Handbook of Scheduling: Algorithms, Models, and Performance Analysis, chapter 15. CRC, 2004. 